\title{
Plumbing Neutron Stars to New Depths with the Binding Energy of the Exotic Nuclide ${ }^{82} \mathrm{Zn}$
}

\author{
R. N. Wolf,,${ }^{1, *}$ D. Beck, ${ }^{2}$ K. Blaum, ${ }^{3}$ Ch. Böhm, ${ }^{3}$ Ch. Borgmann, ${ }^{3}$ M. Breitenfeldt, ${ }^{4}$ N. Chamel,,${ }^{5}$ S. Goriely, ${ }^{5}$ \\ F. Herfurth, ${ }^{2}$ M. Kowalska, ${ }^{6}$ S. Kreim, ${ }^{3,6}$ D. Lunney, ${ }^{7}$ V. Manea,${ }^{7}$ E. Minaya Ramirez, ${ }^{2,8}$ S. Naimi, ${ }^{7,9}$ \\ D. Neidherr, ${ }^{2,3}$ M. Rosenbusch, ${ }^{1}$ L. Schweikhard, ${ }^{1}$ J. Stanja, ${ }^{10}$ F. Wienholtz, ${ }^{1}$ and K. Zuber ${ }^{10}$ \\ ${ }^{1}$ Institut für Physik, Ernst-Moritz-Arndt Universität Greifswald, 17487 Greifswald, Germany \\ ${ }^{2}$ GSI Helmholtzzentrum für Schwerionenforschung GmbH, Planckstraße 1, 64291 Darmstadt, Germany \\ ${ }^{3}$ Max-Planck-Institut für Kernphysik, Saupfercheckweg 1, 69117 Heidelberg, Germany \\ ${ }^{4}$ Instituut voor Kern- en Stralingsfysica, KU Leuven, Celestijnenlaan 200d, B-3001 Heverlee, Belgium \\ ${ }^{5}$ Institut d'Astronomie et d'Astrophysique, CP-226, Université Libre de Bruxelles, 1050 Brussels, Belgium \\ ${ }^{6}$ CERN, 1211 Geneva 23, Switzerland \\ ${ }^{7}$ CSNSM-IN2P3-CNRS, Université Paris-Sud, 91405 Orsay, France \\ ${ }^{8}$ Helmholtz-Institut Mainz, 55099 Mainz, Germany \\ ${ }^{9}$ RIKEN Nishina Center for Accelerator-based Science, RIKEN, 2-1 Hirosawa, Wako-shi, Saitama 351-0198, Japan \\ ${ }^{10}$ Institut für Kern- und Teilchenphysik, Technische Universität Dresden, 01069 Dresden, Germany
}

(Received 28 October 2012; published 22 January 2013)

\begin{abstract}
Modeling the composition of neutron-star crusts depends strongly on binding energies of neutron-rich nuclides near the $N=50$ and $N=82$ shell closures. Using a recent development of time-of-flight mass spectrometry for on-line purification of radioactive ion beams to access more exotic species, we have determined for the first time the mass of ${ }^{82} \mathrm{Zn}$ with the ISOLTRAP setup at the ISOLDE-CERN facility. With a robust neutron-star model based on nuclear energy-density-functional theory, we solve the general relativistic Tolman-Oppenheimer-Volkoff equations and calculate the neutron-star crust composition based on the new experimental mass. The composition profile is not only altered but now constrained by experimental data deeper into the crust than before.
\end{abstract}

DOI: 10.1103/PhysRevLett.110.041101

PACS numbers: 26.60.Gj, 07.75.+h, 21.10.Dr

With the mass of the Sun compressed to the size of an average terrestrial city, neutron stars are among the densest objects known in the cosmos. While there are still many uncertainties, the details of the neutron-star crust composition are of particular interest in view of the possibility that neutron stars contribute to the abundance of lighter elements close to stability and heavier, neutron-rich nuclides that accumulate near shell closures as observed in our Solar System and Galaxy. The mechanism, rapid neutron capture (the $r$ process), has been the subject of intense activity since its first suggestion, but the astrophysical site where it takes place remains a mystery (see review by Arnould et al. [1]). While (type-II) supernovae have been favored for a long time, many problems continue to thwart the correct modeling of an $r$ process. Neutron stars offer a tantalizing alternative $r$-process site because of their large neutron content, a critical ingredient lacking in supernovae models $[2,3]$. As discussed recently [4], the decompression of neutron-star matter brought by tidal effects from a merger with a black hole or another neutron star allows an $r$ process to occur as the ejected clump vaporizes into the interstellar medium. While the ejected mass per event is relatively low, it can still explain the total enrichment of $r$ nuclei in the Galaxy. However, the astrophysical plausibility of this scenario requires a proper understanding of neutron stars, most importantly of the composition of their outer crusts.

As their name implies, these residues of core-collapse (type-II) supernova explosions are essentially composed of neutrons. Three regions can be distinguished (Fig. 1): a locally homogeneous core and two concentric shells, characterized by different inhomogeneous phases [5]. The outermost shell, the so-called "outer crust," consists of a crystal of ionized atoms coexisting with a quantum gas of electrons. While nuclei that are stable under terrestrial conditions are found at the star's surface, those deeper are increasingly neutron-rich. The point where neutrons start to drip out marks the transition to the "inner crust," an assembly of neutron-proton clusters immersed in a sea of unbound neutrons and electrons. Even deeper into the star, the crust dissolves into a uniform liquid of nucleons and leptons until the core is reached.

The conditions prevailing in the deep interior of a neutron star are so extreme that they cannot be reproduced in the laboratory. However, knowledge of specific nuclear binding energies for which high-precision mass measurements are indispensable, combined with neutron-star models, can place the composition of the outer crust on firm ground. Indeed, in analogy with ice cores, it is possible to "drill" into the neutron star and to determine the most abundant species in each layer. In their landmark paper, Baym et al. [6] showed that the only property of relevance (aside from the well-known electron and lattice energy) is the nuclear binding energy. Using known masses, the composition of the outer crust had already been robustly determined to a depth of about $212 \mathrm{~m}$ for a canonical neutron star of 1.4 solar mass and $10 \mathrm{~km}$ radius $[7,8]$. 


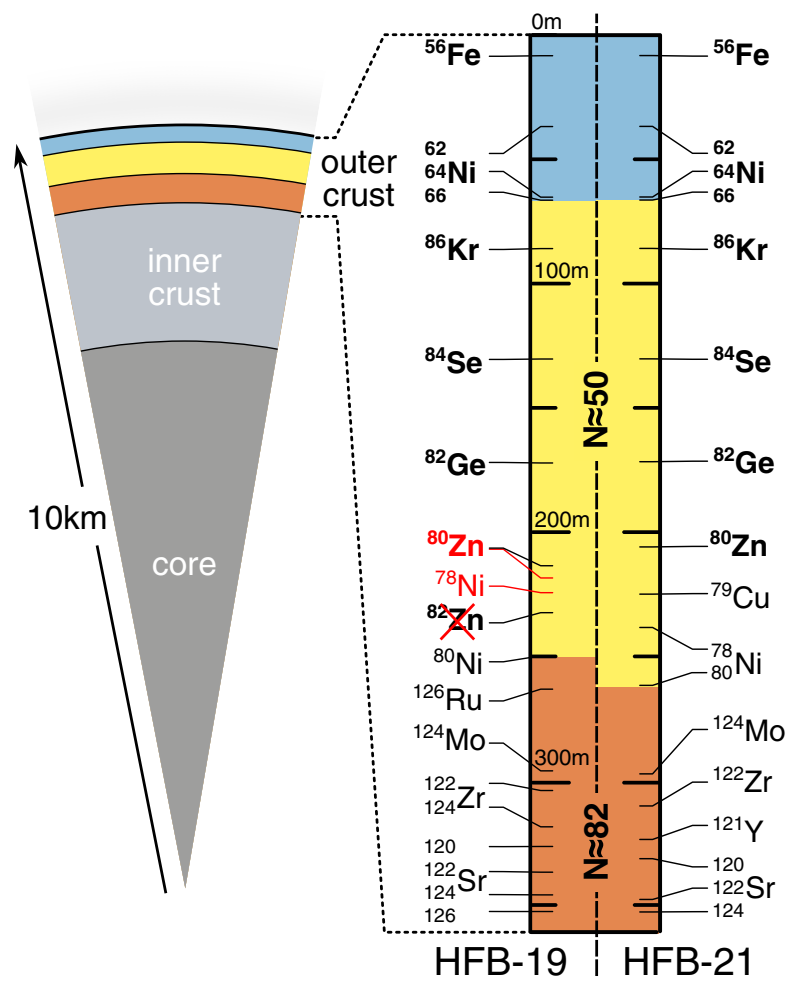

FIG. 1 (color online). The depth profile of a neutron star of 1.4 solar mass and $10 \mathrm{~km}$ radius. The scale on the right indicates the nuclide composition in the outer crust as predicted by the HFB19 and HFB-21 mass models. Experimentally known nuclides are printed in bold. Including the new mass value for ${ }^{82} \mathrm{Zn}$, the position of ${ }^{80} \mathrm{Zn}$ has changed and ${ }^{78} \mathrm{Ni}$ replaces ${ }^{82} \mathrm{Zn}$ (changes marked red).

In this Letter we report the first measurement of the mass of ${ }^{82} \mathrm{Zn}$, which represents the frontier of knowledge for the $N=50$ shell and as such the limit of knowledge for fathoming the neutron-star crust composition.
To plumb a neutron star, Einstein's equations of general relativity, which govern hydrostatic equilibrium, are solved as described by Tolman [9] as well as Oppenheimer and Volkoff [10]. The so-called TOV equations relate pressure and mass-energy density with neutron-star mass and radius. Stable and radioactive-beam facilities have already provided substantial information about a plethora of finite nuclei for the required equation of state (EOS) but even the most neutron-rich of these nuclei still have proton fractions of about $40 \%$, i.e., far larger than the few percent in neutron-star cores or about $30 \%$ at the bottom of the outer crust. To make any statement about the composition of the deeper levels of the outer crust, one has to resort to theoretical mass models and extrapolate from the known masses to nuclei closer to the neutron drip line. However, different state-of-the-art microscopic mass models predict different compositions. They can only be tested by highprecision mass measurements on even more exotic species. The Brussels-Montreal microscopic atomic mass models based on the Hartree-Fock-Bogoliubov (HFB) method have been developed to simultaneously describe the binding energy of exotic nuclides, as well as the EOS of neutron matter resulting from many-body calculations with realistic two- and three-nucleon forces [11]. They can be applied to predict the properties of the inner crust and even of the liquid core, thus providing a consistent and unified treatment of all regions of a neutron star [12].

Mass measurements of exotic nuclides are a topic of intense research pursued at many laboratories worldwide [13-17] as they provide critical information about shell stability and other nuclear-structure effects. Because of nuclear shell effects, the exotic nuclides residing in neutron-star crusts accumulate around the magic neutron numbers $N=50$ and $N=82$, for the latter even at the vicinity of the neutron drip line (see Fig. 2). Binding

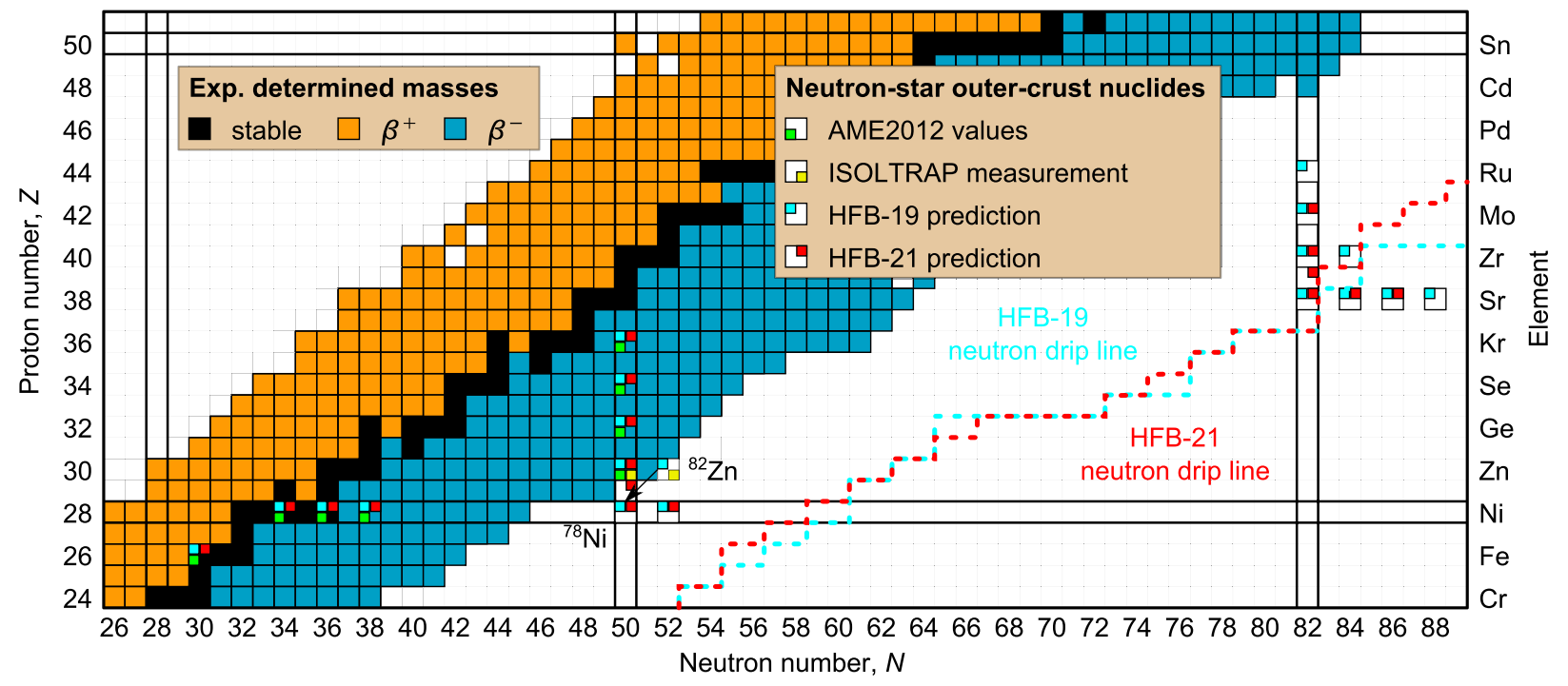

FIG. 2 (color online). The nuclear chart based on AME2012 [38], highlighting the present ISOLTRAP results and the nuclides that compose neutron-star outer crusts according to experiment and the HFB-19 and HFB-21 models. 
energies for the $N=50$ isotones were previously measured down to ${ }^{81} \mathrm{Ga}(Z=31)$ [18], but this nuclide is not predicted to be present in the neutron-star crust (see Fig. 1). In a dedicated experiment that exploited the most advanced techniques for the production and handling of exotic radionuclides, we have measured the $(Z=30){ }^{82} \mathrm{Zn}$ mass. Having the most extreme neutron excess among the known $N=52$ isotones, ${ }^{82} \mathrm{Zn}$ is of particular relevance for the persistence of the strength of the $N=50$ shell-which has been questioned [13] - and thus of critical importance for the prediction of its presence in the neutron-star crust.

The ISOLTRAP Penning-trap mass spectrometer [19] (Fig. 3) at the radioactive-beam facility ISOLDE-CERN [20] has pioneered the art of on-line precision mass measurements. It uses electromagnetic fields to confine ions in an unperturbed environment and to measure their cyclotron frequency. The ISOLDE facility produces zinc isotopes by neutron-induced fission of uranium nuclei. To this end, instead of the uranium-carbide target itself, a tungsten converter [21] was bombarded by a $1.4-\mathrm{GeV}$ proton beam. This technique reduced isobaric contamination, which would result from direct spallation reactions. In addition, a highly selective three-step laser excitation [22] to ionize zinc isotopes was applied as well as a temperature-controlled quartz transfer line [23] that prevented the copious surface-ionized rubidium isotopes from drowning the zinc ion beam. Despite these state-of-the-art precautions, over 6000 ions per second of ${ }^{82} \mathrm{Rb}$ were still present in the ${ }^{82} \mathrm{Zn}$ beam delivered to ISOLTRAP - to be compared to just a few ions of interest. Thus, the previous mass-measurement attempts only reached ${ }^{81} \mathrm{Zn} \mathrm{[24]} \mathrm{but} \mathrm{fell} \mathrm{short} \mathrm{of}{ }^{82} \mathrm{Zn}$ several times at different facilities, making it one of the most challenging nuclides for mass studies to date.

To succeed in measuring the ${ }^{82} \mathrm{Zn}$ mass, yet another type of ion trap was integrated into the ISOLTRAP

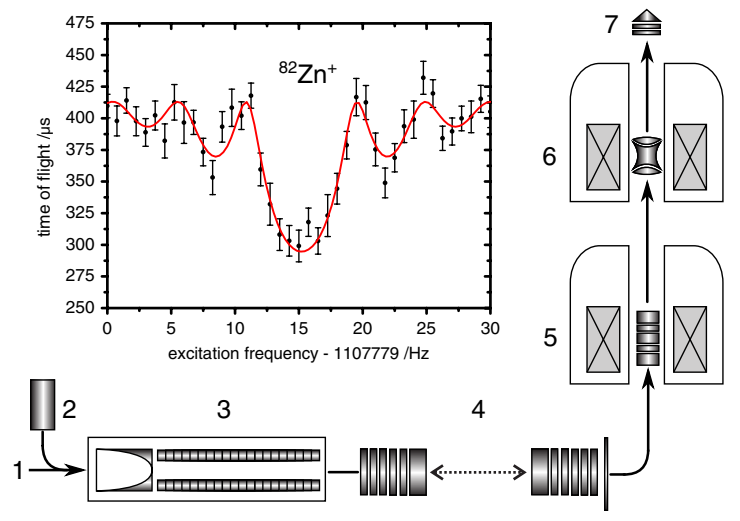

FIG. 3 (color online). Schematic ISOLTRAP overview and time-of-flight resonance of ${ }^{82} \mathrm{Zn}^{+}$. The main components relevant for this study are the incoming ISOLDE beam (1), reference ion source (2), RFQ cooler and buncher (3), MR-ToF mass separator (4), preparation Penning trap (5), precision Penning trap (6), and ToF detector (7). Top-left inset: Time-of-flight resonance of ${ }^{82} \mathrm{Zn}^{+}$(see text). mass-spectrometer setup, previously consisting of a linear Paul trap (radio-frequency quadrupole [RFQ] cooler and buncher [25]) and two Penning traps: A multireflection time-of-flight mass separator (MR-ToF MS) allowed a separation of the residual ${ }^{82} \mathrm{Rb}^{+}$contaminants from ${ }^{82} \mathrm{Zn}^{+}$by repeated oscillations between electrostatic ion mirrors [26,27]. The decisive advantage compared to purification in Penning traps is a mass-resolving power $R_{\mathrm{FWHM}}=m / \Delta m$ in excess of 100000 [28-30] that is obtained in only several tens of milliseconds compared to hundreds of milliseconds in Penning traps. This gain of an order of magnitude in time expands the frontiers of exotic nuclides accessible by ion-trap facilities, as for ${ }^{82} \mathrm{Zn}$ with a half-life of $t_{1 / 2}=228(10) \mathrm{ms}$ [31].

In this first on-line application, the ${ }^{82} \mathrm{Zn}^{+}$ions were sent from the MR-ToF MS through a Bradbury-Nielsen gate [32], which deflected the contaminants. In particular, the radioactive ion beam from ISOLDE was accumulated for $100 \mathrm{~ms}$ after each proton pulse in the RFQ cooler and buncher and stored for additional $5 \mathrm{~ms}$ to thermalize the last incoming ions in a helium buffer-gas environment. The ensemble containing the mixture of ${ }^{82} \mathrm{Zn}^{+}$and ${ }^{82} \mathrm{Rb}^{+}$ isotopes was then injected into the MR-ToF mass separator for 100 revolutions, equivalent to a flight time of about $2.5 \mathrm{~ms}$. This was sufficient to separate the two species by multiple signal widths; i.e., the difference in time of flight was $\approx 500 \mathrm{~ns}$ and the individual signal widths $\approx 200 \mathrm{~ns}$ at the $1 \%$-intensity level. This corresponds to a massresolving power of about $R_{1 \%}=m / \Delta m=6000$, adequate to separate the ${ }^{82} \mathrm{Rb}^{+}$contamination. Subsequently, the ${ }^{82} \mathrm{Rb}^{+}$ions were deflected from the beam-line axis by the Bradbury-Nielsen gate and thus removed from the bunch before injection into the next ion trap. This successful purification of an isobaric radioactive beam was decisive for the present measurement.

The isolated sample was transferred to the first of the two Penning traps situated in individual superconducting solenoids, where the ions were cooled in a helium buffer-gas environment as a preparation for the final mass measurement in the second, hyperbolic high-precision Penning trap. This preparation was accomplished in only $15 \mathrm{~ms}$ by applying a high helium pressure of about $10^{-3}$ mbar. The whole accumulation, purification, and preparation steps were performed in less than $25 \mathrm{~ms}$, which is an order of magnitude faster compared to the commonly used mass-selective buffer-gas centering [33]. In the precision Penning trap, the ions' cyclotron frequency, $\nu_{c}=q B /(2 \pi m)$ in the magnetic field, $B$, was measured via their flight time to a particle detector (time-of-flight ion-cyclotron resonance, ToF-ICR [34]) as a function of the frequency of a radiofrequency (rf) excitation (inset of Fig. 3). To determine the ions' mass $m=r\left(m_{\mathrm{ref}}-m_{e}\right)+m_{e}$, the cyclotronfrequency ratio $r=\nu_{c}^{\mathrm{ref}} / \nu_{c}$ of the ion of interest and a well-known reference isotope to calibrate the magnetic field, here ${ }^{85} \mathrm{Rb}^{+}$, has to be evaluated. 
In total, five measurements could be completed in $16 \mathrm{~h}$ with a total number of 1754 detected ${ }^{82} \mathrm{Zn}^{+}$ions. These experiments included three conventional ToF-ICR measurements, one with $100 \mathrm{~ms}$ and two with $200 \mathrm{~ms}$ rf-excitation time, and two Ramsey-type ToF-ICR resonances [35] with an excitation-waiting-excitation scheme of $20 \mathrm{~ms}-160 \mathrm{~ms}-20 \mathrm{~ms}$. The measurements resulted in a mean frequency ratio for ${ }^{82} \mathrm{Zn}^{+}$and ${ }^{85} \mathrm{Rb}^{+}$of $r=\nu_{c}^{\text {ref }} / \nu_{c}=0.9651728601(391)$ and a mass-excess value of $\mathrm{ME}\left({ }^{82} \mathrm{Zn}\right)=m-A \mathrm{u}=-42.314(3) \mathrm{MeV} / c^{2}$ (where $A$ is the mass number and $\mathrm{u}$ the unified atomic mass unit) with a relative mass uncertainty of $\delta \mathrm{m} / \mathrm{m}=$ $4 \times 10^{-8}$. The latter is dominated by the statistical uncertainty of the cyclotron frequency.

With this new ${ }^{82} \mathrm{Zn}$ mass value, calculations as described in Ref. [8] were performed to drill deeper down into the neutron-star crust. To this end the nuclides which minimize the Gibbs free energy per nucleon were determined as a function of the pressure in the neutron-star crust. By the use of the TOV equations, it was found to which depths in the crust these pressures correspond. The composition profile of a (cold, non-accreting and non-rotating) neutron star of 1.4 solar mass and $10 \mathrm{~km}$ radius was calculated with the new ${ }^{82} \mathrm{Zn}$ mass value and compared to the three most recent Brussels-Montreal mass tables HFB-19, HFB-20, and HFB-21. We have restricted our comparisons to these models because we also use their predictions of the equation of state of neutron-star matter for consistency (few mass models can provide such information). A more extensive study, involving a wide range of mass models, is beyond the scope of this Letter and will be the subject of a future publication. ${ }^{82} \mathrm{Zn}$ is considerably less bound than predicted by HFB-19 and HFB-20 (ME HFB-19,20 $=$ $-42.96 \mathrm{MeV} / c^{2}$ ). From our measurement, ${ }^{82} \mathrm{Zn}$ is no longer present in the neutron-star crust (see Fig. 1). Moreover, the location of ${ }^{80} \mathrm{Zn}$ is limited to a deeper level $(223 \mathrm{~m})$ than predicted by HFB-19. This result has extended the knowledge of the neutron-star crust composition, literally, to new depths.

While HFB-19 (as well as HFB-20) did not correctly predict the new ${ }^{82} \mathrm{Zn}$ mass, the prediction of HFB-21 was close enough $\left(\mathrm{ME}_{\mathrm{HFB}-21}=-42.70 \mathrm{MeV} / c^{2}\right)$ that the predicted neutron-star profile remains the same. Moreover, the fact that HFB-21 is slightly favored is significant in light of another recent mass measurement: that of the neutron star PSR J1614-2230. The measured value of 1.97(4) solar masses [36] excludes many theoretical models for the dense-matter EOS, in particular that of HFB-19, which predicts a lower maximum neutron-star mass [37]. As explained in Ref. [11], the three models HFB-19 to 21 were purposely constrained to reproduce three different representative realistic neutron-matter EOS (as obtained from many-body calculations using realistic two- and three-body forces) up to the highest densities prevailing in neutron stars. It is intriguing that a new mass measurement from nuclear physics is consistent with a new mass from astronomy and that this advance in nuclear theory passes an important test of consistency and accuracy for astrophysical applications.

The authors thank J.M. Pearson, Dépt. de Physique, Université de Montréal, Montréal (Québec), Canada, for fruitful discussions in the preparation of this manuscript. This work was supported by the German Federal Ministry for Education and Research (BMBF) (Grants No. 06GF9102 and No. 06GF9101I), the Max-Planck Society, the European Union seventh framework through ENSAR (Contract No. 262010), the MASCHE project, the French IN2P3, the Nuclear Astrophysics Virtual Institute (NAVI) of the Helmholtz Association, and the ISOLDE Collaboration.

*wolf@uni-greifswald.de

[1] M. Arnould, S. Goriely, and K. Takahashi, Phys. Rep. 450, 97 (2007).

[2] S. Goriely, N. Chamel, H. T. Janka, and J. M. Pearson, Astron. Astrophys. 531, A78 (2011).

[3] L. F. Roberts, D. Kasen, W. H. Lee, and E. Ramirez-Ruiz, Astrophys. J. 736, L21 (2011).

[4] S. Goriely, A. Bauswein, and H.-T. Janka, Astrophys. J. 738, L32 (2011).

[5] J. M. Lattimer and M. Prakash, Science 304, 536 (2004).

[6] G. Baym, C. Pethick, and P. Sutherland, Astrophys. J. 170, 299 (1971).

[7] S. B. Rüster, M. Hempel, and J. Schaffner-Bielich, Phys. Rev. C 73, 035804 (2006).

[8] J. M. Pearson, S. Goriely, and N. Chamel, Phys. Rev. C 83, 065810 (2011).

[9] R. C. Tolman, Phys. Rev. 55, 364 (1939).

[10] J. R. Oppenheimer and G. M. Volkoff, Phys. Rev. 55, 374 (1939).

[11] S. Goriely, N. Chamel, and J. M. Pearson, Phys. Rev. C 82, 035804 (2010).

[12] J. M. Pearson, N. Chamel, S. Goriely, and C. Ducoin, Phys. Rev. C 85, 065803 (2012).

[13] D. Lunney, J. M. Pearson, and C. Thibault, Rev. Mod. Phys. 75, 1021 (2003).

[14] K. Blaum, Phys. Rep. 425, 1 (2006).

[15] Special Issue on Ultra-Accurate Mass Spectrometry and Related Topics, edited by L. Schweikhard and G. Bollen [Int. J. Mass Spectrom. 251, 85 (2006)].

[16] J. Äystö, Nucl. Phys. A805, 162c (2008).

[17] B. Franzke, H. Geissel, and G. Münzenberg, Mass Spectrom. Rev. 27, 428 (2008).

[18] J. Hakala, S. Rahaman, V.-V. Elomaa, T. Eronen, U. Hager, A. Jokinen, A. Kankainen, I. D. Moore, H. Penttilä, S. Rinta-Antila, J. Rissanen, A. Saastamoinen, T. Sonoda, C. Weber, and J. Äystö, Phys. Rev. Lett. 101, 052502 (2008).

[19] M. Mukherjee, D. Beck, K. Blaum, G. Bollen, J. Dilling, S. George, F. Herfurth, A. Herlert, A. Kellerbauer, H. J. Kluge, S. Schwarz, L. Schweikhard, and C. Yazidjian, Eur. Phys. J. A 35, 1 (2008). 
[20] E. Kugler, Hyperfine Interact. 129, 23 (2000).

[21] U. Köster, Eur. Phys. J. A 15, 255 (2002).

[22] V. N. Fedosseev, L.-E. Berg, D. V. Fedorov, D. Fink, O. J. Launila, R. Losito, B. A. Marsh, R. E. Rossel, S. Rothe, M. D. Seliverstov, A. M. Sjödin, and K. D. A. Wendt, Rev. Sci. Instrum. 83, 02A903 (2012).

[23] E. Bouquerel, R. Catherall, M. Eller, J. Lettry, S. Marzari, and T. Stora, Nucl. Instrum. Methods Phys. Res., Sect. B 266, 4298 (2008).

[24] S. Baruah, G. Audi, K. Blaum, M. Dworschak, S. George, C. Guénaut, U. Hager, F. Herfurth, A. Herlert, A. Kellerbauer, H.-J. Kluge, D. Lunney, H. Schatz, L. Schweikhard, and C. Yazidjian, Phys. Rev. Lett. 101, 262501 (2008).

[25] F. Herfurth, J. Dilling, A. Kellerbauer, G. Bollen, S. Henry, H. Kluge, E. Lamour, D. Lunney, R. Moore, C. Scheidenberger, S. Schwarz, G. Sikler, and J. Szerypo, Nucl. Instrum. Methods Phys. Res., Sect. A 469, 254 (2001).

[26] H. Wollnik and M. Przewloka, Int. J. Mass Spectrom. Ion Process. 96, 267 (1990).

[27] W. R. Plaß, T. Dickel, U. Czok, H. Geissel, M. Petrick, K. Reinheimer, C. Scheidenberger, and M. I. Yavor, Nucl. Instrum. Methods Phys. Res., Sect. B 266, 4560 (2008).

[28] R. N. Wolf, M. Eritt, G. Marx, and L. Schweikhard, Hyperfine Interact. 199, 115 (2011).

[29] R. N. Wolf, G. Marx, M. Rosenbusch, and L. Schweikhard, Int. J. Mass Spectrom. 313, 8 (2012).
[30] R. N. Wolf, D. Beck, K. Blaum, Ch. Böhm, Ch. Borgmann, M. Breitenfeldt, F. Herfurth, A. Herlert, M. Kowalska, S. Kreim, D. Lunney, S. Naimi, D. Neidherr, M. Rosenbusch, L. Schweikhard, J. Stanja, F. Wienholtz, and K. Zuber, Nucl. Instrum. Methods Phys. Res., Sect. A 686, 82 (2012).

[31] M. Madurga et al., Phys. Rev. Lett. 109, 112501 (2012).

[32] N.E. Bradbury and R. A. Nielsen, Phys. Rev. 49, 388 (1936).

[33] G. Savard, S. Becker, G. Bollen, H.-J. Kluge, R. Moore, T. Otto, L. Schweikhard, H. Stolzenberg, and U. Wiess, Phys. Lett. A 158, 247 (1991).

[34] G. Gräff, H. Kalinowsky, and J. Traut, Z. Phys. A 297, 35 (1980).

[35] S. George, S. Baruah, B. Blank, K. Blaum, M. Breitenfeldt, U. Hager, F. Herfurth, A. Herlert, A. Kellerbauer, H.-J. Kluge, M. Kretzschmar, D. Lunney, R. Savreux, S. Schwarz, L. Schweikhard, and C. Yazidjian, Phys. Rev. Lett. 98, 162501 (2007).

[36] P. B. Demorest, T. Pennucci, S. M. Ransom, M.S.E. Roberts, and J.W.T. Hessels, Nature (London) 467, 1081 (2010).

[37] N. Chamel, A. F. Fantina, J. M. Pearson, and S. Goriely, Phys. Rev. C 84, 062802(R) (2011).

[38] M. Wang, G. Audi, A. Wapstra, F. Kondev, M. MacCormick, X. Xu, and B. Pfeiffer, Chinese Phys. C 36, 1603 (2012). 